Article

\title{
The Mach's Principle and large-scale modification of Newto- nian gravitation as alternative approach to Cold Dark matter and Dark Energy
}

\author{
Nelson Falcon ${ }^{1, *}$
}

1 Universidad de Carabobo Dpto. de Física FACYT. Venezuela; nelsonfalconv@gmail.com
* Correspondence: nelsonfalconv@gmail.com; Tel.: +58 4124099940

\begin{abstract}
We take a heterodox approach to the $\Lambda$ FRW Cosmology starting from the modification of Newtonian gravity by explicitly incorporating Mach's Principle through an additional term a great scale in the gravitation. The results show that at the after of the matter-radiation decoupling, the distribution of matter at scales greater than $10 \mathrm{Mpc}$ contributes with an inverse Yukawa-like field, which verifies the observations: resulting null in the inner solar system, weakly attractive in ranges of interstellar comoving distances, very attractive in comoving distance ranges comparable to the clusters of galaxies, and repulsive in cosmic scales. This additional term explains dark energy, removes the incompatibility between the density of matter and the flatness of the universe; and also allows the theoretical deduction of the Hubble-Lemaitre Law. Additionally, Birkhoff Theorem, Virial Theorem, the missing mass of Zwicky, the BAO, gravitational redshift are discussed. It is concluded that the dark energy and the missing mass can be approached with the usual physics if a classical, large-scale modification of the Inverse Square Law.
\end{abstract}

Keywords: Mach's Principle; Dark Matter; Dark Energy; gravity; graviton mass; $\Lambda$ FRW cosmology; Hubble-Lemaitre Law.

\section{Mach Principle and Phenomenology}

In the book "The Science of Mechanics", Ernst Mach [1] concludes that the local inertial frame is determined in some way by the movement of distant astronomical objects. This conclusion, known as Mach's Principle, arises from the very exact coincidence of the two ways of measuring the rotational speed of the angular velocity of the Earth: dynamically (Foucault's Pendulum) and astronomically (with respect to the "Fixed stars"), as indicated in the figure 1. This coincidence, together with Newton's well-known "bucket experiment", according to which the curvature of a rotating bucket with water is an expression of inertia ("vis insita" in Newton's words).

Inspired by these empirical facts, E. Mach, postulates those inertial forces owe their existence to the distribution of distant matter in the Universe. In other words, the water in Newton's bucket would not bend if there were no galaxies and it is the teleconnection that exactly equates the period of Foucault's pendulum at the North Pole with the sidereal day. As a result, if Newton's bucket was still on the Earth, and put the Universe in its entirety to rotate, it would also bend the surface of the water. Mach's thought decisively influenced Einstein's thought as he himself refers in his autobiographical notes [2] "It was Ernst Mach who with his History of Mechanics ... exerted a profound influence on me ... also during my young years, Mach's epistemological position influenced me a lot, a position that today seems essentially untenable".

The General Theory of Relativity (TRG) when connecting gravitational fields with inertia, seem to give entry to Mach's Principle, through the Field Equations. But they do not fully verify it, because the limit conditions of the Field Equations. From Einstein's 
point of view, the "relativity of inertia" was an integral part of his General Theory [3], that is to say: the inertial field defined by the metric tensor $\left(g^{\mu \nu}\right)$ had to be completely determined by the distribution of masses and energies in the Universe $\left(T^{\mu v}\right)$; but this is not completely true because the relationship established by the Field Equations are differential and it is necessary to impose limit conditions at infinity to unequivocally determine the $g^{\mu v}$ from a given $T^{\mu v}$. This need to impose additional conditions prompted the incorporation of the cosmological constant in Einstein's Model of the Universe, which we now incorporate through the Friedmann-Robertson-Walker models with cosmological constant ( $\Lambda$ FRW model).

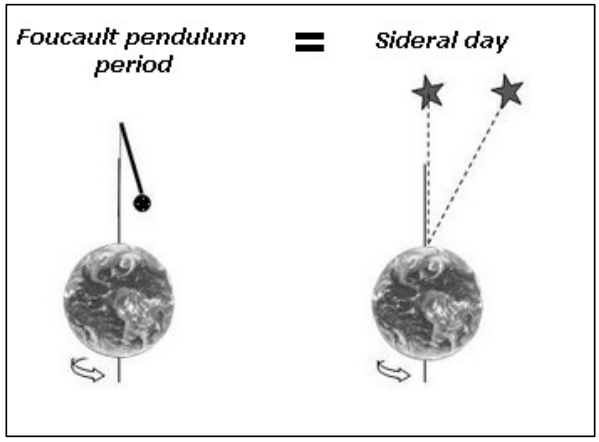

Figure 1. Illustration of Mach's principle: an Foucault pendulum period, in north pole, is exactly an sidereal day (Own source).

If local inertia is somehow linked to the large-scale distribution of matter in the Universe, then the inverse square law of Newtonian gravitation is insufficient to describe it. The gravitational field at any point in our galaxy or at any other point in space (a point in the local group of galaxies for example) would be the sum of the gravitational contributions of all the other galaxies at that point; and although these contributions were insignificant, their total sum is not necessarily is null. So the gravitational interaction between two stars would be the one prescribed by inverse-square Newton's Law plus an additional contribution from the distant masses, which does not have to be the same for different points in space; since, on a large scale, the distribution of masses is not spherical with its center in the local frame of observation.

By other hand, assuming that the dynamics of the universe is prescribed only by the Newton law of gravity we encounter serious difficulties in describing the dynamic of the Universe [4]: (i) It cannot explain the rotation curves of galaxies, which show its incompatibility with the virialized masses (ii) the mass observed into the rich clusters of galaxies is significantly less than that required to maintain these systems gravitationally stable, and (iii) In cosmological scales, the observed baryonic matter density is much lower than predicted by the FRW models with cosmological constant. The problem of missing mass appears to affect the dynamics at all length scale beyond the Solar System. One solution has been to propose the requirement of missing matter of unknown origin (non baryonic Dark Matter) with equally unknown properties and only interacts gravitationally with ordinary matter. However, after more than a two decades of strenuous efforts: theoretical, astronomical observations and laboratory experiments, only their existence has been suggested conjectural or paradigmatically.

It is worth asking, then, if any modification of the Law of Gravitation is possible that on a large scale solves the dynamics observed in the universe (including cosmic acceleration: Dark energy), and also, agrees with the certainties of Newtonian gravitation. Such a modification of gravitation should agree, at the scale of the solar system, with the inverse-square law and be compatible with terrestrial experiments (Eövos-like experiment).

The purpose of this work is to deduce a possible modification of the Law of Gravitation that meets these requirements, that is, that all particles with nonzero rest mass, is 
subject gravitational inverse-square law, plus an additional term that varies with the comoving distance. This complementary contribution to the inverse-square law, would be caused by distribution at great scale of the baryonic mass, in the sense of Mach's principle. This inverse Yukawa-like Potential [4,5], would be null in very near solar system, slightly attractiveness in ranges of interstellar distances, very attractiveness in distance ranges comparable to galaxies cluster and repulsive to cosmic scales .

For this, the mean energy per nucleon during the decoupling between material and radiation in the early universe is discussed (section II) and the form of the Yukawa-like potential is deduced together with the coupling constants, consistent with the previously reported parameters $[5,6]$. The implications of Birkhoff's theorem and the general form of the potential are shown in section III. The cosmological and astrophysical implications are summarized in section IV, together a preliminary study of its implications in the Baryonic Acoustic Oscillations (BAO) and the Cosmic Microwave Background (CMB), before the conclusions in the last section.

\section{Matter-radiation decoupling}

During recombination, in the first moments of the formation of the Universe, when the material is separated from radiation and hydrogen is synthesized (surface of last scattering), the average energy per unit mass $(U)$ can be expressed for each nucleon a the temperature $\mathrm{T}$ of the plasma, using the Boltzmann distribution, as the work required to move the proton from the initial position to the comoving distance $r$ relative to the center of the protogalactic cloud of mass $M$, as :

$$
\langle\mathrm{U}\rangle \equiv\left\langle\frac{\mathrm{u}}{\mathrm{N}}\right\rangle=-\mathrm{U}_{\mathrm{o}}\left(\mathrm{r}_{\mathrm{o}}-\mathrm{r}\right) \exp \left(-\frac{\varepsilon}{\mathrm{k}_{\mathrm{B}} \mathrm{T}}\right)
$$

Where $k_{B}$ denotes the Boltzmann constant, $\mathrm{U}_{\mathrm{o}}$ is an constant and $\varepsilon$ is the proton energy. This energy is only kinetic energy, which could be expressed in terms of the gravitational energy of the protogalactic clouds that forms later, thus

$$
\varepsilon=\frac{m_{p} v^{2}}{2} \cong \frac{1}{2} \frac{G m_{p} M}{r}
$$

Where, $\mathrm{m}_{\mathrm{p}}$ is the rest-mass of proton. Notice that we can use Virial theorem in its usual form, because we are at $z=1100$ and the first protostars and protogalaxies that appear much later, around $z=6$, have not yet formed.

Using (1) and (2), then

$$
U_{Y F}(r) \equiv\langle\mathbf{U}\rangle=\mathbf{U}_{\mathbf{0}}\left(r-r_{0}\right) e^{-\alpha / r}
$$

With

$$
\alpha=\frac{G m_{p}}{k_{B} T_{d}} M=\frac{4 \pi G m_{p}}{3 k_{B} T_{d}} r_{0} \rho_{c}
$$

Where $T_{d}$ is the average plasma temperature at the moment of the decoupling $\left(k_{B} T_{d \approx 13,6 \mathrm{eV}}\right) . M$ and $r_{0}$ is the mass and average radius of protogalaxy respectively, and $\rho_{c}$ is the critical density. $U_{o} \equiv U_{o}(M)=4 \pi l \mathrm{GM} \mathrm{r}_{0}^{-1}$ is a coupling constant in units of $J / k g \quad\left(l \equiv 1 \mathrm{~m}^{-1}\right.$ is a dimensional parameter $)[5,6]$ because it is the gravitational potential in upper limit when $\varepsilon / T \rightarrow 0$ in the protogalaxy of mass $M$.

By other hand, the energy balance in the protogalaxy demands that

$$
k_{B} T_{d}=\frac{G m_{p}}{r_{0}} M
$$

Because the contribution of the large scale of gravitation (UYF) is null in $r \approx r_{0}$. Remember that $U=U_{N}+U_{Y F}$, i.e. the total gravitational energy is the Newton gravity plus the large scale contribution (UYF-field). 
Using the critical density [4-6] $\rho_{c} \cong 9.5(8 \pi)^{-1} 3 H_{0}{ }^{2} G^{-1} \approx 1.8610^{12} M_{\odot} / M p c^{3}$ in (5), through the model of Modification of the Newtonian Dynamics, we obtain $r_{0}=47.12 \mathrm{Mpc}$; and replacing this value in (4) we obtain $\alpha \cong 2.47 M p c$. Notice that these values are closely close to the values that adjust the phenomenology of the from the astronomical data ro 50 Mpc and $\alpha \sim 2.5 \mathrm{Mpc}$ [5] . Thus we can write (3) with $r$ in $M p c$, as:

$$
U_{Y F}(\operatorname{rin} M p c) \cong \mathbf{U}_{\mathbf{0}}(r-50) e^{-2.5 / r}
$$

Also, the force per unit mass (acceleration), complement to large-scale of the Newtonian gravitation is :

$$
F_{I Y}(r) \equiv-\vec{\nabla} U_{Y F}=-\frac{U_{0}(M)}{r^{2}} e^{-\alpha / r}\left(r^{2}+\alpha\left(r-r_{0}\right)\right)
$$

Note that FIY is null by $r_{\mathrm{c}}$

$$
r_{c}=\frac{\alpha}{2}\left(\sqrt{1+\left(\frac{4 r_{0}}{\alpha}\right)}-1\right) \cong 9.62 M p c \approx 10 M p c
$$

as the previous report [5,6], according to the assumption to the finite range of the gravitation; i.e. with massive graviton [5]:

$$
m_{g}^{0} \cong \frac{\hbar}{r_{c} c} \approx 10^{-64} \mathrm{~kg} \simeq 10^{-29} \frac{\mathrm{eV}}{\mathrm{c}^{2}}
$$

And the maximum $r_{\mathrm{m}}$ occurs in

$$
r_{m}=\frac{\alpha r_{0}}{2 r_{0}+\alpha} \cong 1.203 M p c \approx 1.2 M p c
$$

This value is in order of the Abell radius, for the typical clusters of galaxies; as the previous assumption [5]. We can see in (7), that if $r$ is negligibly small compared to $r=2.5$ $M p c$, then $F_{I \gamma} \approx 0$, i.e. the gravity interaction is only prescribed by the inverse square law of gravitation, in accordance with Eövos-like experiments.

For ranges the comoving distances, between objects gravitationally bound, with $r$ smaller we obtain $F_{I Y} \sim r^{-1}$, see for details [5], therefore, the MoND-Milgrom results are recovered as a particular case, and could be applied to solve the problem of the rotation curves of galaxies.

\section{Birkhoff's theorem and Overview}

The general solution to the gravitation Poisson equation, under spherical symmetry, depends on the mass distribution outside of $r$ :

$$
U(r)=-4 \pi G\left[\frac{1}{r} \int_{0}^{r} \rho\left(r^{\prime}\right) r^{\prime 2} d r^{\prime}+\int_{r}^{\infty} \rho\left(r^{\prime}\right) r^{\prime} d r^{\prime}\right]
$$

In the Newtonian gravity approach it is easily understood that the second term in (11) is canceled because of the fact that the solid angles extending from one point within a sphere to opposite directions have areas in the sphere that escalade as $r^{2}$, while The gravitational force per unit the dough scales such as $r^{-2}$, so that the gravitational forces of the two opposing areas are canceled exactly. The equivalent in general relativity is called 
Birkhoff's theorem. But in general approach, if the gravitation field has a large scale contribution, then:

$$
U(r)=U_{N}+U_{Y F}=-\frac{G M}{r}+U_{0}(M)\left(r-r_{0}\right) e^{-\alpha / r}
$$

Thus, it's no true that the second term in (11) are null. Therefore, Birkhoff's theorem could not be applied in the renormalized Newtonian theory of gravitation, such as in the UYF-field.

The figure 2 shown the second term of the (12), i.e. the UYF given in (3), for different ranges of comoving distance $r$, and the phenomenological description.

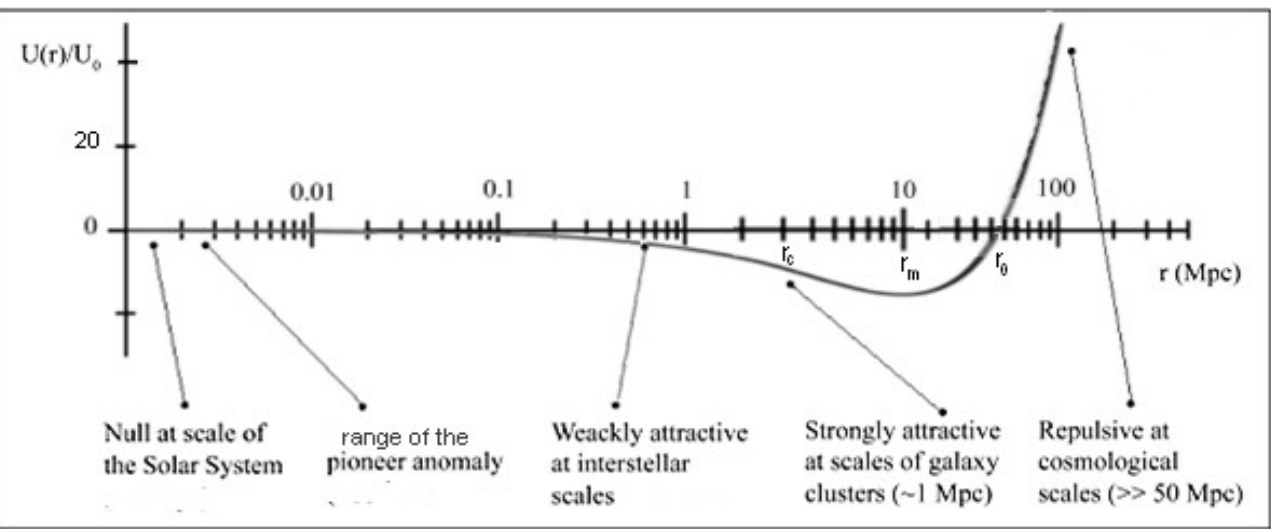

Figure 1. The Yukawa-like potential, complementary contribution to the inverse-square law, in comoving scale, at several astronomical ranges (Own source).

. Note that the potential per unit mass (UYF) results in a smooth function: continuous, differentiable, approaching zero for small range of comoving distance $r$. The fig. 2 show that UYF has a unique minimum, for the comoving distance in the order of $10 \mathrm{Mpc}$, as can be expected if the graviton has non-zero rest mass, in according to the detection of gravitational waves [7] i.e. it suggests that the scope of the gravitational force is finite.

Newton's law of gravitation implicitly postulates that the range of the force is infinite and consequently, the force would have a greater range than the universe itself, besides, of course, it assumes null rest mass for the graviton. Also, an infinite range of gravity would imply that large-scale structures in the Universe would be spherically symmetric. However, the distribution of hot gas in the superclusters of galaxies, detected via the Suyaev-Zel'dovich effect [8], and the large-scale structures in the distribution of galaxies, with dimensions greater than $10 \mathrm{Mpc}$ : as e.g. Sloan Great Wall and the Voids, , do not show symmetric axial distribution [9]. A finite range of gravity (on the order of $10 \mathrm{Mpc}$ ) can explain these structures by a chain sequence of gravitational attractions between their neighboring components and not necessarily by a common center, of colossal masses in the superclusters of galaxies (i.e.: black super holes). It is clear that until today no accretion disks of Super Black Holes, have been detected, despite of advances in X-ray and IR astronomy.

The UYF is null in the r-values near the average distance between galaxy clusters $\left(r \approx r_{0} \sim 50\right.$ $M p c$ ). Besides, this function has only one inflection point; i.e. their derivative (force by mass units) is maximum in $r$-value in order of the nucleus of Abell's radius $\left(r=r_{c} \approx 1.2 \mathrm{Mpc}\right)$. And also its derivative (acceleration), is constant at cosmological scales (r>>50 Mpc) such as it would be required to incorporate the cosmological constant $(\Lambda)$.

As example, in figure 3 is plotted the effective gravitational energy, per unit mass, for various members of the local group of Galaxies, (Equation 12). In the left panel, for very close satellite gal- 
axies (in logarithmic scale). In the right panel, to show other notable members, of the Local Group, on a linear scale. The galaxy VV124 (UGC4879) maybe the most isolated dwarf galaxy in the periphery of the Local Group, near of the minimum of gravitational energy in accord with present description. Also M3, M33, NGC 300 and NGC55 they have a gravitational potential energy per unit mass, 100 times greater than the spheroid satellite galaxies of the Milky Way. The consequences of this, in dynamic stability and in the description of the rotation curves, is outside the scope of this communication, and would be interesting as an additional test for the approach to the large-scale modification of inverse square law of Newtonian gravity.

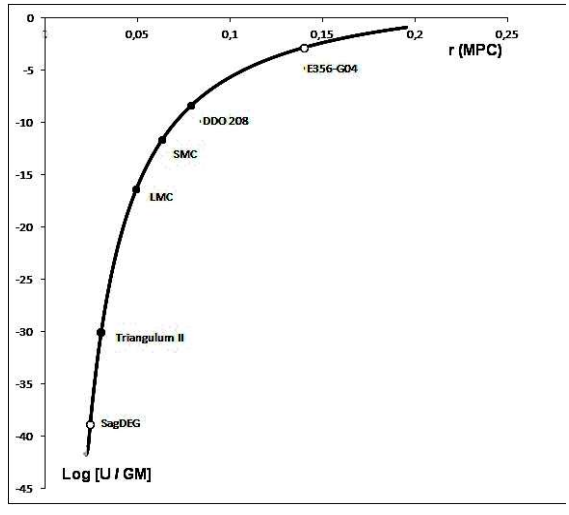

(a)

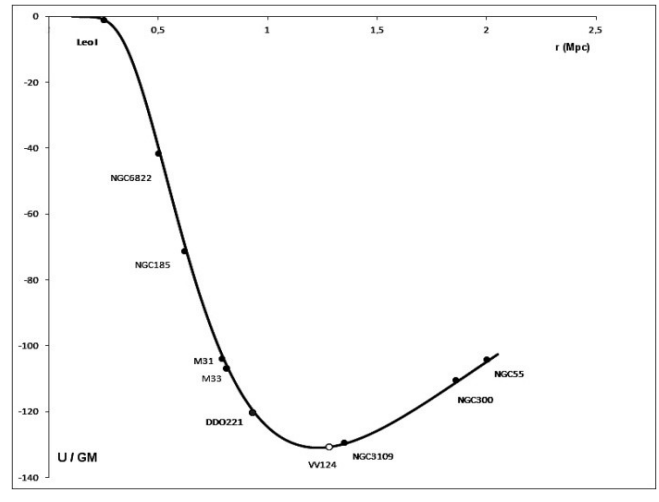

(b)

Figure 3. Gravitational Energy by mass unit in galaxies of Local Group (Equation 12). (a) Very near galaxies satellites of Milky Way, on logarithmic scale. (b) Galaxies of the Local group, on linear scale. (Own source).

\section{Cosmological and astrophysics consequences}

We consider a model of the expanding universe, where each galaxy is a particle of a perfect fluid, under the hypotheses of homogeneity and isotropy; i.e. a $\Lambda$ FRW cosmological model. The cosmological term leads to usual Friedmann equations [4,5]:

$$
\begin{aligned}
& \left(\frac{\dot{R}(t)}{R(t)}\right)^{2}+\frac{k c^{2}}{R^{2}(t)}=\frac{8 \pi G}{3} \rho+\frac{\Lambda c^{2}}{3} \\
& \frac{2 \ddot{R(t)}}{R(t)}+\left(\frac{\dot{R}(t)}{R(t)}\right)^{2}+\frac{k c^{2}}{R^{2}(t)}=-\frac{8 \pi G}{c^{2}} P+\Lambda c^{2}
\end{aligned}
$$

In this approach the Dark Energy can be thought of as a "cosmic acceleration" ( the cosmological constant as an force by unit of mass):

$$
\Lambda=\Lambda_{0} F_{I Y}(r)=-\Lambda_{0} \frac{d U_{Y F}}{d r}
$$

Where the coupling constant is

$$
\Lambda_{0} \equiv \frac{3 H_{0}}{c^{3}} \cong 0.3110^{-42} \mathrm{~m}^{-3} \mathrm{~s}^{2}
$$




\section{1 interpreting the cold dark matter}

When $r \rightarrow r_{m}$, the comic parameter $\Lambda$ in the intergalactic scale is

$$
\Lambda\left(r_{m}\right) \simeq 10.55\left(4 \pi G \mathrm{~kg} \mathrm{~m}^{-2}\right) \frac{3 H_{0}}{c^{3}}
$$

We obtain $[5,6]$ in flat universe model $(k=0)$ :

$$
\begin{aligned}
& 0=H_{0}{ }^{2}\left[\left(\Omega_{b}+\Omega_{b} \Omega_{I Y}\right)+\Omega_{\Lambda}-1\right]=H_{0}{ }^{2}\left[\Omega_{m}+\Omega_{\Lambda}-1\right] \\
& q_{0}=\frac{\left(\Omega_{b}+\Omega_{b} \Omega_{I Y}\right)}{2}\left(1+\frac{3 P}{c^{2} \rho}\right)-\Omega_{\Lambda}=\frac{\Omega_{m}}{2}\left(1+\frac{3 P}{c^{2} \rho}\right)-\Omega_{\Lambda}
\end{aligned}
$$

where it has been used the standards cosmological parameters: $\Omega_{b}=\rho / \rho_{c}, \Omega_{\Lambda}=$ $\Lambda c^{2} / 3 H_{0}^{2}, \mathrm{q}_{0}=-\ddot{R} / R H_{0}^{-2}$ for the density of matter, cosmological term and deceleration respectively; and the definition $\Omega_{\mathrm{IY}} \equiv \Lambda\left(r_{c}\right) c^{2} / 3 H_{0}^{2}$.

Now, the remarkable result in (18) is that the cold dark matter parameter $\left(\Omega_{c}\right)$ could be interpreted as the gravitational contribution caused by the large scale distribution of the ordinary baryonic matter: $\Omega_{m}=\left(\Omega_{b}+\Omega_{b} \Omega_{I Y}\right)=\Omega_{b}+\Omega_{c}$ without postulating the existence of unknown exotic particles (non baryonic dark matter). So, as $\Omega_{I Y} \approx 10.42$ and using $\Omega_{b} \approx 0.0223$ and $\Omega_{\Lambda} \approx 0.6911$ [10], then: $\Omega_{m} \approx 0.255$ and $\Omega_{m}+\Omega_{\Lambda}=0.255+0.6911 \approx 1$.

\subsection{About the Dark Energy}

When $r \rightarrow r_{c}$, the comic parameter $\Lambda(r)$ in the intergalactic scale, is using (15):

$$
\Lambda\left(r_{c}\right) \cong 0.623\left(4 \pi G \mathrm{~kg} \mathrm{~m}^{-2}\right) \frac{3 H_{0}}{\mathrm{c}^{3}}=0.623 \frac{3 H_{0}^{2}}{\mathrm{c}^{2}}
$$

Thus, in the present model, the dark energy would be the cosmic acceleration in local frameworks, caused by the large scale distribution of the ordinary baryonic matter, as prescribed the Mach's Principle, through of $U_{Y F}$ proposed. As before, replacing (20) in cosmological density parameter then (see [5] for detail):

$$
\Omega_{\Lambda} \equiv \frac{\Lambda\left(r_{c}\right) c^{2}}{3 H_{0}^{2}} \cong 0.623 \frac{\left(4 \pi G k g m^{-2}\right)}{H_{0} c} \approx 0.623 h^{-1}
$$

Using here the upper limit for Hubble parameter $h=0.863$ [6], we obtain $\Omega_{\Lambda} \approx 0.72$ in good agreement with the measurements of type Ia Supernovae.

\subsection{The Hubble-Lemaître's Law and the Age Universe}

Consider the photons emitted from a remote galaxy with recession velocity $v$, and their observation in the reference local frame. Therefore, we should evaluate (3) at $r>>$ $50 M p c$, with initial condition $v=0$ in $t=0$. We find: 


$$
V=\int a d t=\int\left(\lim _{x \rightarrow \infty} F_{I Y}(x)\right) \frac{d r}{c} \simeq \frac{U_{0}}{c} r
$$

Replacing as before, $\bigcup_{0}=4 \pi \mathrm{G} \mathrm{kg} \mathrm{m}^{-2}$, we obtain de Hubble-Lemaître's Law [4-6] :

$$
V=\left(\frac{4 \pi G \ell}{c}\right) r \equiv H_{0} r \cong\left(86,3 \frac{k m}{s M p c}\right) r
$$

Where $\ell \equiv 1 \mathrm{~kg} \mathrm{~m}^{-2}$ is a dimensional parameter.

Notice that de value of $\mathrm{H}_{0}$ is the theoretical upper limit, evaluate for most distant objects $(r>>50 \mathrm{Mpc})$. Most recent direct measurements of the constant of Hubble which Space Telescope (HST), are $H_{0}=75.8^{+5.2}-4.9 \mathrm{~km} \mathrm{~s}^{-1} \mathrm{Mpc}^{-1}$ and $78.5^{+6.3}-5.8 \mathrm{~km} \mathrm{~s}^{-1} \mathrm{Mpc}^{-1}$ depending of the target calibration [11], furthermore Riess [12] had found that $H_{0}=74.22 \pm 1.82 \mathrm{~km} \mathrm{~s}^{-1}$ $\mathrm{Mpc}^{-1}$ in the very near Large Magellanic Cloud (LMC), also $\mathrm{H}_{0} \approx 83.56 \mathrm{~km} \mathrm{~s}^{-1} \mathrm{Mpc}^{-1}$ in a selected sample of 392 galaxies, in a range of 50 - $1400 \mathrm{Mpc}$ [6]. The results of the Planck Collaboration [10] throw lower values for $\mathrm{H}_{0}$. In the Planck Collaboration, the estimates of the cosmological parameters, show degeneration in the simultaneous estimates of $\mathrm{H}_{0}$ and $\Omega \mathrm{m}$; because the CMB measurements are not a direct measure of the Hubble constant.

Also, the Friedmann equation can be written as:

$$
\tau=H_{0}^{-1} \int_{0}^{\infty}\left[(1+z)^{3}\left(\Omega_{b}+\Omega_{b} \Omega_{I Y}\right)+\Omega_{\Lambda}\right]^{-\frac{1}{2}} \frac{d z}{z+1}
$$

Note that it is the $\Lambda F R W$ conventional equation for the age of universe, because of arithmetic equality: $\left(\Omega_{b}+\Omega_{b} \Omega_{I Y}\right)=\Omega_{b}+\Omega_{c}$. The numerical integration in the present model $\left(\Omega_{\mathrm{b}} \approx 0.0223, \Omega_{\mathrm{IY}} \approx 10.42 \Omega_{\Lambda} \approx 0.623\right.$ and upper limit of $\left.\mathrm{H}_{0} \approx 86,3 \mathrm{~km} \mathrm{~s}^{-1} \mathrm{Mpc}^{-1}\right)$ gives that the age of the universe is $\tau \sim 11.42 \mathrm{Gyr}$, in good agreement with the age of the White

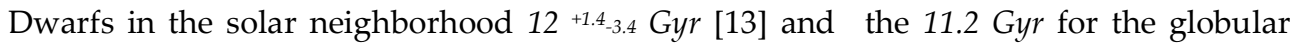
clusters in the Milky [14]. The direct Cosmochronology through White Dwarfs, offer an independent technique for the age of Milky Way, while that using Planck collaboration data-set $\left(\Omega_{\mathrm{b}} \approx 0.0223, \Omega_{\Lambda} \approx 0.677\right.$ and $\left.\mathrm{H}_{0} \approx 67.74\right)$ we obtain $\tau \sim 13.8$ Gyr [10].

\subsection{The missing mass of Zwicky}

Let us consider the Clausius's Virial expression $G=\sum_{i} \vec{p}_{i} \vec{r}_{i}$ and deriving with respect to time, and then averaging with respect to a complete period $(\tau)$, we obtain the well-known virialized expression between kinetic energies and power. But now, the gravitational potential energy is given by (12) then [5] :

$$
\langle U\rangle=2\langle T\rangle+\frac{4 \pi G M r_{0}^{-1} l}{\tau} \int_{0}^{\tau} \sum_{i} m_{i} e^{-\alpha / r}\left(\alpha+r_{0}-\frac{\alpha r_{0}}{r}\right) d t^{\prime}=2\langle T\rangle+4 \pi G M l r_{0}^{-1} \alpha \sum_{i} m_{i}
$$

An important result is that (25) solve the Zwicky's paradox. The "missing mass" could be interpreted as the energy associated with the UYF field. For example in the particular case of the Coma cluster then:

$$
\left.G M 4 \pi l r_{0}^{-1}\left[e^{-\alpha / r}\left(r-r_{0}\right)+\alpha\right]\right|_{r=4.71} \approx 710^{19} m^{2} s^{-2} \gg \frac{v^{2}}{2} \simeq 1.1210^{14} \mathrm{~m}^{2} \mathrm{~s}^{-2}
$$


The introduction of the UYF changes the Kepler's third law, taking the time derivative of (12) then:

$$
\frac{4 \pi^{2}}{\tau^{2}}=\frac{G M}{r^{3}}-\frac{U_{0}(M)}{r^{3}} e^{-\alpha / r}\left(r^{2}+\alpha\left(r-r_{0}\right)\right)
$$

Where $\tau$ denote the orbital period.

This result can also be used to reinterpret the rotation curves as the MoND-Milgrom theories [4] where the velocity dispersion increases monotonically with radial distance instead of the Keplerian behavior predicted by the inverse square law of Newtonian gravitation.

\subsection{Arp Controversy and Gravitational redshift.}

The photons emitted by the Newtonian potential source (GM / $R$ ) would also be affected by the local contribution of the gravitational field produced by the large-scale distribution of matter, then the gravitational component of the redshift $(\mathrm{zg})$, in term of the gravitational potential $\phi$, is:

$$
z_{g}=-\frac{\phi}{c^{2}}=-\frac{1}{c^{2}}\left(-\frac{G M}{R}+U_{Y F}\right)=\left[\frac{R_{s}}{2 R}\right]\left[-\frac{1}{R}+4 \pi l e^{-\alpha / r} \frac{r-r_{0}}{r_{0}}\right]
$$

Where use the Schwarzschild's radius: $R_{s}=2 G M / c^{2}$.

The figure 4 show the Gravitational redshift for compact objects, note that that in distance ranges of the order of one third of the range of gravity the gravitational redshift remains constant for any compact object considered, therefore, the observation of these objects outside the local group of galaxies will allow the cosmological redshifts to be calibrated.

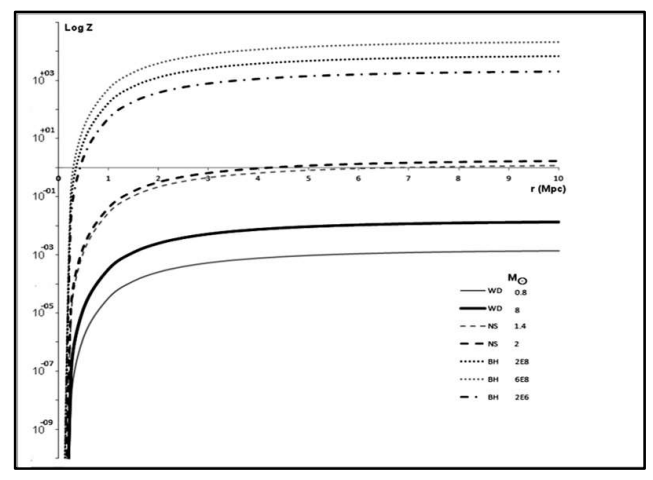

Figure 4. Gravitational redshift in compact objects vs. comoving distance. (Own source).

An important result in (28) is that two galaxies an equal distance could be present redshift different, thus it's solvent the Arp's controversy [15]. Observe that second term in (28) increase the Gravitational redshift until 7.83 and would help understand the problem of AGN at High-redshift and the nature of Quasars. 


\subsection{Gravitational Lensing}

Let us consider the deflection of a point-like lens of mass $\mathrm{M}$, under the assumption of basic thin gravitational lent, when gravitational potential is small, the effect of the space-time curvature on light trajectory can be described as an effective refraction index $\eta$, given by:

$$
\eta=1-\frac{2}{c^{2}} \Phi \cong 1+\frac{2}{c^{2}} \Phi
$$

The deflection angle $(\zeta)$ of the light rays which traveling in a gravitational field is given by the integration of the gradient component of $\eta$ orthogonal to the trajectory:

$$
\vec{\zeta}=-\int \vec{\nabla}_{\perp} \eta d l=\frac{2}{c^{2}} \int \vec{\nabla}_{\perp} \Phi d l
$$

But now, the gravitational field $\Phi$ is given (12), assuming that is small, i.e. $\alpha / r<<1$ we obtain:

$$
\Phi=U(r)=U_{N}+U_{Y F} \cong-\frac{G M}{r}+\frac{4 \pi G M \alpha_{0}}{r}
$$

Where, we used $\alpha_{0} \cong \alpha / \mathrm{r}_{0}$, and $U_{o}(M)=4 \pi l G M r_{0}^{-1}$ as before.

If $\mathrm{b}$ is the impact parameter of the unperturbed light ray and $y$ denote the position along the unperturbed path as measured from the point of minimal distance from the lent: $r^{2}=y^{2}+b^{2}$,

$$
\vec{\varsigma}=\frac{2}{c^{2}} \int \vec{\nabla}_{\perp} U d z=\frac{4 G M}{c^{2} b} \hat{b}-\frac{4 \pi G M}{c^{2} b} \alpha_{0} \hat{b}
$$

Where we using previous notation, $R_{P}$ denote the physical radius of stars and $R_{s}$ is the Schwarzschild radius, we find the deflection angle:

$$
\varsigma \approx \frac{2 R_{S}}{3 c^{2} R_{P}}
$$

Note that the inclusion of the potential (12) leads to a reduction of one third in the calculation of the angle of deflection of the gravitational Lensing. Consequently, estimates of the deflecting mass in gravitational lenses observed at long distances (where the large-scale correction for Newtonian gravitation makes sense) would have been underestimated by a factor of three. Obviously, these do not affect the observation of the deflection of light, in the case of a total solar eclipse, because the $U_{Y F}$ is null at the scale of the solar system (figure 2) because comoving range of distance is much smaller than kiloparsecs.

\subsection{Longitude and Mass of Jean}

A self-gravitating, proto-stellar or proto-galactic mass, remains in hydrodynamic equilibrium as long as its dimensions do not exceed the length of Jeans. The Jean's length $\left.(\lambda]^{0}\right)$ is the characteristic length for which pressure balances gravity; which in term of the density $(\rho)$, temperature $\left(T_{\mathrm{e}}\right)$ and hydrogen mass $\left(m_{H}\right)$ is: 


$$
\lambda_{J}^{0} \equiv \frac{c_{s}}{t_{s}} \simeq \frac{c_{s}}{t_{g}}=\sqrt{\frac{3 \pi k_{B} T_{e}}{G \rho m_{H}}}
$$

The effective gravitational free-fall collapse is the Newtonian potential plus the contribution of UYF. Replacing (27) into (34), given that $t g \approx \tau$ then

$$
\lambda_{J}=\lambda_{J}^{0}\left[1-\frac{3 M l r_{0}^{-1}}{\rho} r^{-3} e^{-\alpha / r}\left(r^{2}+\alpha\left(r-r_{0}\right)\right)\right]^{-1 / 2}
$$

Consider a uniform self-gravitating mass, with electronic temperature and effective density, constants. In the Newtonian Gravitation approach, the Jeans length $\left(\lambda_{J}^{0}\right)$ remains constant for all $r$ and therefore agglutination and / or fragmentation will be homogeneous. Figure 5 shows that, when considering a large-scale modification of Newtonian gravitation, through the potential $U_{Y F}$, the effective Jeans length $\left(\lambda_{J}\right)$ decreases for the mega parsec range, reaching a minimum of approximately half the Abell radius favoring the agglutination.

The reason for this is that the gravitational potential now exerts a stress on the gas, between the center (where the term Newtonian $\mathrm{r}^{-2}$ dominates) and the periphery (where the term of the force due to $U_{Y F}$ dominates) whose maximum is in order of Abell: 1.2 Mpc.

As the Jeans Masses, is proportional to the cube of Jeans longitude, would be decrease very much. The Jeans mass is the critical mass above which gravity dominates, so if the Jeans Mass decreases then the gravity dominates, and so a perturbation will collapse even further when compressed, leading to run-away collapse in range of comoving distance of the megaparsec.

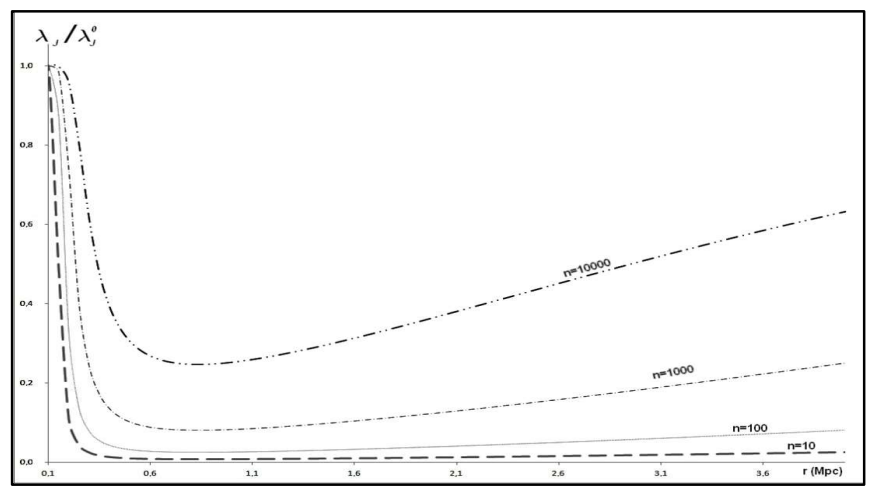

Figure 5. Jean's length corrected for potential UYF vs comoving distance in scale of the clusters of galaxies, for several number density (Own source).

\subsection{Angular Diameter distance}

The angular diameter distance is, using (24) and the redshift dependence of universe-scale factor:

$$
D_{A}=\frac{c H_{0}^{-1}}{z+1} \int_{0}^{z}\left[(1+z)^{3} \Omega_{b}\left(1+\Omega_{I Y}\right)+\Omega_{\Lambda}\right]^{-\frac{1}{2}} d z
$$


In Fig. 6, we shown the dimensionless angular diameter with and without UYF (dashed lines), and only the parameter of the matter density (continues line: $\Lambda=0$ ). We can see that the UYF reduces the angular diameter distance for all z-values and their maximum in $\mathrm{z} \approx 2$.

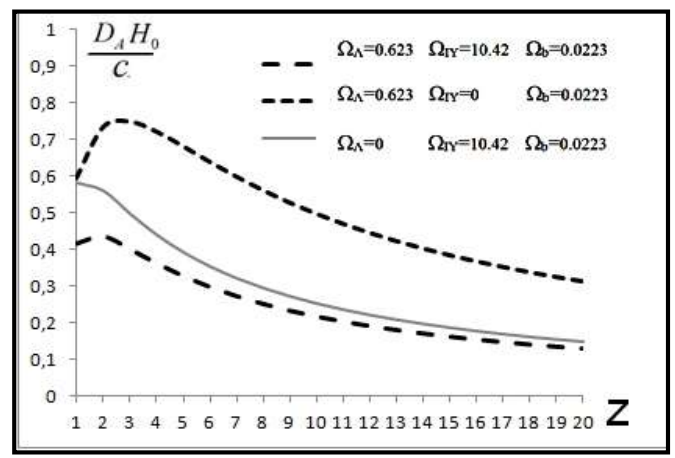

Figure 6. Angular diameter distance in function of the redshift [5]

\section{$4.9 B A O$ and $C M B$ anisotropies}

The early universe consisted of hot plasma of photons, electrons and baryons closely coupled by Thomson scattering, with oscillations in the photon fluid, due to radiation pressure and gravity. The essential physics of these linear perturbations can be study from non-relativistic hydrodynamic approach, through equations of continuity, Euler's and Poisson's equations, in Lagrangian form. Now consider a small perturbation (first order) superimposed on the "background" fluid, then the fractional density perturbation (or density contrast) $\delta \cong \delta \rho / \rho_{0}$ obeys the relations [16]:

$$
\frac{d^{2} \delta}{d t^{2}}+2 H \frac{d \delta}{d t}+\left[c_{S}^{2} k_{S} R^{-2}(t)-4 \pi G \rho_{0}\right] \delta=0
$$

Where $R(t)$ is the scale factor, $H$ is the Hubble parameter, $c_{s}$ the sound speed and $k_{s}$ is a comoving wavenumber of a plane-wave disturbance.

Notice that the UYF field is not taken into account in Poisson's equation, because the disturbances occur at the moment of decoupling long before the formation of the protostars and therefore there is not yet the distribution of masses due to the inertial frame, referred to by the Mach principle represented by the UYF field.

In a $\Lambda$ CDM model, the growing modes of the time-dependence perturbations [17] is given by the growth function:

$$
G(z)=\frac{5 \Omega_{m}}{2} H(z) \int_{z}^{\infty} H^{-3}\left(z^{\prime}\right)\left(1+z^{\prime}\right)^{3} d z^{\prime}
$$

Where the Hubble parameter is given by Friedmann equation (24), and wan be write as:

$$
H(z)=\left[(1+z)^{3} \Omega_{m}+(1+z)^{4} \Omega_{r a d}+\Omega_{\Lambda}\right]^{1 / 2}
$$

In usual formalist of growth of structure and galaxies formation, beginning by the oscillations acoustic adiabatic (BAO), it's used $\Omega m=\Omega_{b}+\Omega_{c}$ in (37) and (38). It's easy see that the large-scale modification of Newtonian gravitation as alternative approach to cold dark matter would given an identical result for $\mathrm{BAO}$, by the arithmetic identity: $\Omega_{m}=\Omega_{b}+\Omega_{c}=\Omega_{b}\left(1+\Omega_{Y_{F}}\right)$ as previous discussion in section 4.1 and 4.3, i.e. interpreting the term referring to cold dark matter without invoking the existence of non-baryonic exotic matter. 
In the present $U_{Y F}$-field formalist the dependence of density and pressure remain unchanged on the scale factor of expansion $R(t)$, and therefore, not affects the Early Universe. Thus, during the decoupling matter-radiation, the primordial fluctuations and the anisotropies in the $\mathrm{CMB}$, the nucleosynthesis (baryogenesis), should remain unchanged by a large-scale modification of Newtonian gravity. Remark that UYF is forty orders of magnitude higher than the average distance per nucleon in the primordial plasma. Also the Sachs-Wolfe effect also does not change, because the size of the horizon at the time of recombination is approximately $100 \mathrm{kpc}$, much less than the maximum range of the gravitational force with massive graviton $(\sim 10 \mathrm{Mpc})$ and at such ranges, the graviton would travel the entire universe inside the horizon without decay.

\section{Summaries and Conclusion}

The inverse square law of Newtonian gravity implicitly assumes a null mass for the graviton and an infinite range of gravitational force, which contradicts the finiteness of the observable universe and the existence of gravitational waves. If local inertia is somehow linked to the large-scale distribution of matter in the Universe (Mach`s Principle), then the inverse square law is insufficient to describe it. Also it's cannot explain the rotation curves of galaxies, neither the missing mass of Zwichy, and is not enough for to explain the observed baryonic matter density in cosmological scales. So in $\Lambda$ FRW cosmological models to appear the paradigmatically concept about non baryonic Dark Matter (cold dark matter).

By other hand, using the Boltzmann distribution, the calculus of the mean energy per nucleon during the surface of last scattering, leads to at complementary contribution to the Newton gravity. This inverse Yukawa-like field (UYF) would be caused by distribution at great scale of the baryonic mass, in the sense of Mach's principle. This UYF-field result: null in very near solar system, slightly attractiveness in ranges of interstellar distances, very attractiveness in distance ranges comparable to galaxies cluster and repulsive to cosmic scales

The consequences of adding a UYF-field as a large-scale contribution of Newtonian gravity [5] implies that:

1. The cold dark matter could be interpreted as the gravitational contribution caused by the large scale distribution of the ordinary baryonic matter: $\Omega_{m}=\left(\Omega_{b}+\Omega_{b} \Omega_{I Y}\right)=\Omega_{b}+\Omega_{c}$

2 The dark energy would be caused by the cosmic acceleration in local frameworks, by the large scale distribution of the ordinary baryonic matter, as prescribed the Mach's Principle

3 The theoretical upper limit for Hubble constant leads $H_{0} \propto \frac{4 \pi G}{c}$, then the age of the universe results in order of the $\sim 11.42 \mathrm{Gyr}$, with very-good agreement with the White Dwarfs Cosmo-chronology.

4 The Virial theorem and Kepler's third law now including an additional term (FYF) that solve the Zwicky's paradox, so the "missing mass" is reinterpreted as the energy of the UYF-field, and rotation curves of galaxies problem could be modeled similarly to the Milgrom models.

5 In the approach of the UYF-field the gravitational component of the redshift increase the gravitational redshift until an factor of $\sim 8$, it's solvent the Arp's controversy, and would help understand the problem of AGN at High-redshift and the nature of Quasars.

6 The inclusion of the UYF-field leads to a reduction in the calculation of the angle of deflection of the gravitational lensing so the deflecting mass at long distances would have been underestimated by a factor of three, without affect the deflection in total eclipses of sun.

7 The UYF reduces the angular diameter distance for all $z$-values with a maximum in $z \approx 2$, in angular diameter distance distribution. 
8 In range of comoving distance of the megaparsec, the Jeans Mass decreases then a perturbation will collapse even further when compressed, leading to run-away collapse of the protogalaxies..

9 The large-scale modification of Newtonian gravitation given an identical result for $\mathrm{BAO}$ as the paradigm of cold dark matter, by the arithmetic identity: $\Omega_{\mathrm{m}}=\Omega_{\mathrm{b}}+\Omega_{\mathrm{c}}=$ $\Omega \mathrm{b}\left(1+\Omega_{\mathrm{YF}}\right)$; but it does not require assuming the existence of non-baryonic exotic particles

10 The CMB and primordial nucleosynthesis remain unchanged by a large-scale modification of Newtonian gravity, such as the UYF-field discussed here.

Regardless of whether the UYF-Field is exactly the one proposed here or not, is that, perhaps, to understand the dynamics of the universe, we would have to abandon the assumption about the universal validity of the inverse square law of Newtonian gravity, assumed uncritically, instead of conjecturing unobservable exotic matter

Funding: "This research received no external funding".

Acknowledgments: We appreciate the understanding and solidarity of the Editors given the particular situation of Venezuelan scientists.

Conflicts of Interest: "The authors declare no conflict of interest."

\section{References}

1. Mach, E. The Science of Mechanics, Cambridge University Press. London.UK $\mathbf{1 8 9 3}$

2. Einstein, A. “Autobiographical Notes" In "Albert Einstein: Philosopher-Scientist”, Paul Schilpp Ed., Illinois USA 1970 pp 17

3. Bondi, H. Cosmology. Cambridge University Press. London UK 1951

4. Falcon, N. Modification of the Newtonian Dynamics in AFRW-Cosmology an alternative Approach to Dark Matter and Dark Energy. JMP 2013, 4, 10-18.

5. Falcon, N A large-scale heuristic modification of Newtonian gravity as an alternative approach to dark energy and dark matter. J Astrophys Astron 2021, 42, 102.

6. Falcon, N.; Aguirre, A. Theoretical Deduction of the Hubble Law Beginning with a MoND Theory in Context of the $\Lambda F R W-C o s m o l o g y$. IJAA 2014, 4, 551-559.

7. LIGO Scientific Collaboration and Virgo Collaboration. GW170104: Observation of a 50-Solar-Mass Binary Black Hole Coalescence at Redshift 0.2. Physical Review Letters. 2017, 118 (22): 221101.

8. Planck Collaboration Planck 2015 results: XXII. A map of the thermal Sunyaev-Zeldovich effect. A \& A 2016, 594, A22

9. Peebles, P. J. E. The Large-Scale Structure of the Universe. Princenton press: N.J. USA 2020

10. Planck Collaboration. Planck 2018 results. VI. Cosmological parameters 2019 arXiv:1807.06209 astro-ph.CO.

11. de Jaege, T. et al. A measurement of the Hubble constant from Type II supernovae. MNRAS 2020, 496 (3).

12. Riess, A. G. Large Magellanic Cloud Cepheid Standards Provide a 1\% Foundation for the Determination of the Hubble Constant and Stronger Evidence for Physics beyond $\Lambda$ CDM. ApJ 2019, 876 (1)

13. Kilic, $\mathrm{M}$ et al The Ages of the Thin Disk, Thick Disk, and the Halo from Nearby White Dwarfs. ApJ 2017, 837 (2)

14. Krauss, L et al. Age Estimates of Globular Clusters in the Milky Way: Constraints on Cosmology . Science 2003, 299: 5603, 65-69

15. Arp, H. Catalogue of Discordant Redshift Associations. Aperion: NJ. USA 2003

16. Peacock, J.A. Cosmological Physics, University Press: Cambridge. UK 1999

17. Bassett, B.A., Hlozek, RBaryon Acoustic Oscillations 2009 arXiv:0910.5224v1 . [astro-ph.CO] 